\title{
Prognostic Significance of Hes-1, a Downstream Target of Notch Signaling in Hepatocellular Carcinoma
}

\author{
Jing-Huai Zou, Tong-Chun Xue, Chun Sun, Yan Li, Bin-Bin Liu, Rui-Xia Sun, \\ Jie Chen, Zheng-Gang Ren, Sheng-Long Ye*
}

\begin{abstract}
Background: Hairy and enhancer of split 1 (Hes-1) protein is a downstream target of Notch signaling and is a basic helix-loop-helix transcriptional repressor. However, definitive evidence for a role in hepatocellular carcinoma (HCC) cells has not been reported. Here, Hes-1 was revealed to an important component of the Notch signaling cascade in HCC cell lines possessing different potential for lung metastasis. Materials and Methods: RNAi mediated by plasmid constructs was used to analyze the role of Hes-1 in MHCC-97L HCC cells by assessing proliferation, apoptosis, cell migration and matrigel invasion following transfection. Hes-1 protein expression analysis in HCC tissue was also conducted by immunohistochemistry. Results: Our studies revealed that Hes-1 was decreased in HCC cell lines with higher lung metastasis potential at both the mRNA and protein levels. Down-regulation of the Hes-1 gene in MHCC-97L cells resulted in increased cell proliferation, reduced apoptosis and increased migration and invasion. Conclusions: Hes-1 has potential prognostic value in post-surgical HCC patients and may be an independent prognostic indicator for overall survival and tumor recurrence. These findings have important implications for understanding the mechanisms by which Hes-1 participates in tumor proliferation and invasion.
\end{abstract}

Keywords: Hepatocellular carcinoma - lung metastasis - notch-1 - Hes-1 - Shh

Asian Pac J Cancer Prev, 16 (9), 3811-3816

\section{Introduction}

Hepatocellular carcinoma (HCC) is the third leading cause of cancer death worldwide, and the second in China (Beasley, 1988; Caporaso et al., 1991). HCC almost always develops in association with chronic hepatitis virus infection or liver cirrhosis (Colombo, 1999; He et al., 2005; Parkin et al., 2005). Diagnosis of HCC carries an extremely poor prognosis because of the high frequency of tumor recurrence or distant metastasis following surgical resection (Portolani et al., 2006). Nevertheless, surgery remains the best current treatment for HCC. The rate of HCC recurrence and/or metastasis following radical resection is $61.5 \%$ for large tumors and 35.4-43.5\% for small tumors (Tang et al., 1999; Mo et al., 2003). Multiple genes and factors are likely involved in the processes underlying HCC recurrence and metastasis, and elucidating their specific molecular mechanisms could aid the search for effective novel therapies and improve outcomes for HCC patients.

Reports have indicated that Notch signaling is involved in HCC carcinogenesis. Deregulation of Notch receptors and/or ligands with subsequent aberrant activation of Notch signaling has been found in a series of malignant tumors
(Lindsell et al., 1995; Ranganathan et al., 2011). Hairy and enhancer of split 1 (Hes-1) protein is a downstream target of Notch signaling and is a basic helix-loop-helix transcriptional repressor (Dailey et al., 2013; Kaushik et al., 2014). Despite the increase in Notch-1 and Hes-1 gene expression that has been reported in HCC samples (Cantarini et al., 2006), the relationship of Notch-1 and Hes-1 protein expression with overall survival (OS) and time to recurrence (TTR) of HCC patients has never been reported. Furthermore, no definitive investigations have reported the influence of Hes-1 in HCC cell lines.

In this study, we have studied Notch-1 and Hes-1 expression patterns in several HCC cell lines with varying metastatic capacity, and examined the influence of Hes-1 expression in a HCC cell line. We have also investigated the correlation of Hes-1 protein expression levels with OS and TTR of HCC patients following surgical resection.

\section{Materials and Methods}

\section{Cell culture}

The human HCC cell lines used in this study (MHCC97-L (low metastatic potential), MHCC97-H and HCCLM3 (both with high metastatic potential)) were 
established at the Liver Cancer Institute, Zhongshan Hospital, Fudan University, Shanghai, China (Tian et al., 1999). Hep3B (very low metastatic potential) was obtained from American Type Culture Collection, Manassas VA. Cells were cultured in Dulbecco's modified Eagle's medium (DMEM; Gibco), supplemented with $10 \%$ fetal bovine serum (FBS; GIBCO) and were incubated at $37^{\circ} \mathrm{C}$ in a humidified atmosphere of $5 \% \mathrm{CO}_{2}$.

RNA purification, notch signaling pathway RT2 profiler PCR array, real-time PCR

Total RNA was prepared using the RNeasy Mini Kit (Qiagen), converted into double-stranded cDNA, and used for RT-PCR array (APHS-059A, SuperArray Bioscience Corporation, Frederick, MD) analysis or quantitative RT-PCR. For cDNA synthesis, $1.5 \mu \mathrm{g}$ of RNAs was synthesized using random hexamers and reverse transcriptase according to the manufacturer's protocol (Invitrogen). Real-time PCR reactions were performed using a Mastercycler ep realplex PCR system (Eppendorf) according to the manufacturer's recommendations.

All mRNA expression levels were normalized to the expression level of GAPDH RNA in all quantitative RT-PCR experiments. The specific primers used were as follows: Notch-1: 5'-CAGGCAATCCGAGGACTATG-3' and 5'-CAGGCGTGTTGTTCTCACAG-3'; Hes1: 5' - TGGATGCGGA G TCTACGATG - 3' and 5'-TAAGGCCACTTGCCACCT TC-3'; GAPDH: 5'-AGCCACATCGCTCAGACA-3' and 5'-GCCCAATACGACCAAATCC-3'.

\section{Western blot analysis}

Protein was extracted from the cell lysate and separated by electrophoresis on $12 \%$ SDS-polyacrylamide gels. Proteins were then transferred for $2 \mathrm{~h}$ at room temperature to polyvinylidene difluoride membranes (Millipore) at $50 \mathrm{~mA}$ using a Tris-glycine buffer system. Membranes were blocked for $2 \mathrm{~h}$ in a solution of $10 \%$ milk powder in $0.1 \%$ Tween-20 in TBS (TBS-T) before primary antibodies against Notch-1 and Hes-1 (both used at 1:2000; Epitomics) were added and membranes were incubated overnight at $4^{\circ} \mathrm{C}$. After washing with TBS-T, the secondary antibody (KPL 1:5000) was added and incubated for $2 \mathrm{~h}$ at room temperature was conducted. Following membrane washing, proteins were visualized with BeyoECL Plus (Beyotime Institute of Biotechnology, Haimen, China).

\section{RNA interference}

The vector constructs pGCSIL-GFP-shRNA-Hes-1 were produced by Shanghai Genechem Company Ltd., Shanghai, China. Three individual shRNA-Hes-1 vectors (pGCSIL-GFP-shRNA-Hes-1) were produced to silence the expression of Hes-1 in MHCC-97L cells (shRNA- Hes1- MHCC-97L). Three shRNA targeting sequences for Hes-1 were used: 1) 5'-TGCATGACCCAGATCAATG-3', 2) 5'-ACGTGCGAGGGCGTTAATA-3', and 3) 5'-TCATCAAAGCCTATTATGG-3', and were designated $8897,8898,8899$, respectively. The mock shRNA lentiviral construct (LV-GFP) was used was control.

Transfection of shRNA constructs into MHCC-
97L cells was performed using Lipofectamine 2000 (Invitrogen) according to the manufacturer's instructions. Stable transfectant clones were selected by addition of $2.0 \mu \mathrm{g} / \mathrm{ml}$ puromycin (Invitrogen) and characterized by quantitative RT-PCR and immunoblotting to determine the expression levels of Hes-1 mRNA and protein.

\section{Cell proliferation, apoptosis, cell migration and matrigel invasion assays}

Cells were aliquoted into a 96 -well plate $\left(2.0 \times 10^{3} /\right.$ well) and incubated for $12 \mathrm{~h}$ at $37^{\circ} \mathrm{C}$ under $5 \% \mathrm{CO}_{2}$. Mock control (LV-GFP) and shRNA-Hes-1 (8897 and 8898) and treated with Hes-1 shRNA for $6 \mathrm{~h}$. After replacing the medium with $100 \mu \mathrm{L}$ DMEM containing $10 \%$ FBS, $10 \mu \mathrm{L} \mathrm{CCK}-8$ solution (Dojindo) was added at indicated time points and plates were incubated for a further $2 \mathrm{~h}$. The absorbance at $570 \mathrm{~nm}$ was measured to determine the number of viable cells in each well. All experiments were performed a minimum of three times.

Apoptosis was measured using an Annexin V-APC/7AAD kit accordance with the manufacturer's protocols (KeyGen Biotech, Nanjing, China). Briefly, cells were re-suspended in the binding buffer provided and reacted with $5 \mu \mathrm{l}$ annexin V/FITC reagent and $5 \mu \mathrm{l}$ of propidium iodide for $15 \mathrm{~min}$ at room temperature in the dark. Stained cells were analyzed by flow cytometry (BD FACSCalibur, BD Biosciences, Bedford, MA).

Cell migration and matrigel invasion analysis was performed using a Transwell (Corning, NY). Forty-eight hours after transfection with RNA interference constructs, the filters coated with matrigel (BD Biosciences) in the upper compartment were coated with $100 \mu \mathrm{L}$ of medium containing $1 \times 10^{5}$ cells, and the lower compartment was filled with $500 \mu \mathrm{L}$ of conditioned culture medium, which was mixed with DMEM supplemented with $10 \%$ FBS. After 36 hours, cells that had migrated to the bottom surface were fixed with ethanol neutral formalin Giemsa stained for counting. All experiments were performed at least three times.

\section{Patients and follow-up}

Tumor specimens used in this study were chosen from 108 HCC patients who underwent liver resection in the Liver Cancer Institute, Zhongshan Hospital, Fudan University between January 2000 and July 2004. Patients were subjected to follow up every three months until March 2010. The median follow-up period was 58.5 months (range: 2-122 months). All patients were prospectively monitored by serum AFP assay, abdominal ultrasonography, and chest X-ray every 1-6 months depending on the postoperative time. Acomputed tomography scan of the abdomen was performed every six months. OS was defined as the time interval between surgery and death or last follow up. The TTR was calculated from the date of tumor resection until the detection of tumor recurrence, death or the last observation.

The study methodologies conformed to the standards set by the Declaration of Helsinki. Ethical approval was obtained from the Zhongshan Hospital Research Ethics Committee and written informed consent from each 
patient were obtained.

\section{Immunohistochemistry and score evaluation}

Tissue microarrays were constructed as reported previously(Jin et al., 2013). Using 108 specimens, tissue cylinders with a diameter of $1.0 \mathrm{~mm}$ were punched from the marked areas of each block and incorporated into a recipient paraffin block. Sections $4-\mu$ m thick were placed on slides coated with 3-aminopropyltriethoxysilane. Paraffin sections were deparaffinized in xylene and rehydrated through decreasing concentrations of ethanol (100\%, 95\%, and 85\%, 5 min each). Antigens were unmasked by microwave irradiation for three min in $\mathrm{pH}$ 6.0 citric buffer and cooled at room temperature for 60 min. Endogenous peroxidase activity was blocked by incubation of the slides in $3 \% \mathrm{H}_{2} \mathrm{O}_{2}$ /phosphate-buffered saline, and nonspecific binding sites were blocked with goat serum. Primary antibodies against Notch-1 and Hes-1 (both used at 1:2000; Epitomics) were used for immunohistochemistry. An EnVision detection kit (GK500705: Gene Tech, Shanghai, China) was used to visualize tissue antigens. Tissue sections were counterstained with hematoxylin for five min. Negative control slides omitting the primary antibodies were created for all assays. Immunohistochemical staining was assessed by two independent pathologists without knowledge of patient characteristics. The immuno-scoring procedure was as follows: the staining intensity was first scored ( 0 , negative; 1 , weak; 2 , moderate; 3 , high) and then the percentage of positive cells was scored $(0,10 \%$ positive cells; $1,10-25 \%$ positive cells; $2,26-50 \%$ positive cells; $3,>50 \%$ positive cells). The final score of each sample was obtained by addition of both staining and percentage scores. Samples were classified as negative when the final

scores were $0(-)$ and when 1-2 as weakly positive (+), 3-4 as moderately positive $(++)$, and more than 5 as strongly positive $(+++)$. For statistical analysis $(-)$ and $(+)$ were defined as low expression, while $(++)$ and $(+++)$ were defined as high expression.

\section{Statistical analysis}

Statistical analyses were carried out with SPSS 12.0 software (SPSS, Chicago, IL, USA). Quantitative variables were analyzed by Student's t test or MannWhitney test. Experimental data were presented as the mean of each condition \pm SD or SEM and $p<0.05$ was considered statistically significant. Univariate and multivariate analyses were based on a Cox proportional hazard regression model. The variables showing significance $(p<0.05)$ by univariate analysis were adopted when multivariate Cox proportional hazards analysis was performed.

\section{Results}

Screening of notch expression profiles in HCC cell lines with different lung metastasis potential

We first screened the expression of Notch factors in MHCC97-L (low metastatic potential) and HCCLM3 (high metastatic potential) cell lines using the Human Notch Signaling Pathway RT2 profiler PCR Array. Six upregulated and twelve downregulated genes of interest were identified. Of these, eight genes had significant differences in expression $(p<0.05)$. The upregulated genes were ccnd1, hes-1, notch-1, and nr4a2, while fzd1, hoxb4, notch-3, and SHH were down-regulated (Figure 1A). Relative expression ratios are shown in Figure 1B, C.

We next performed Notch-1, Hes-1 mRNA and

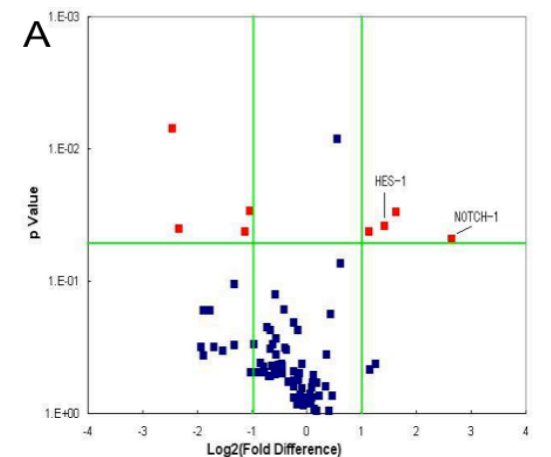

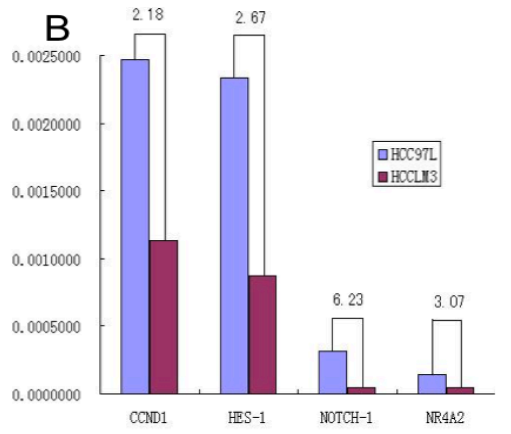

F

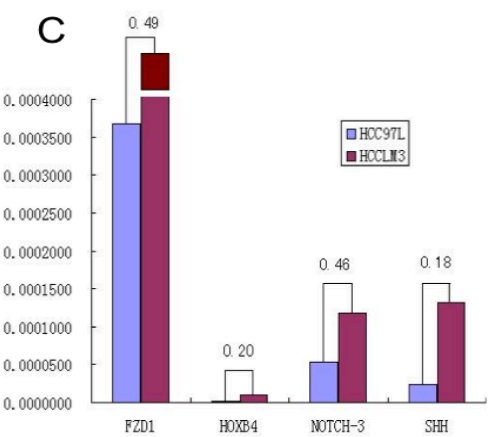

E

$\mathrm{D}$

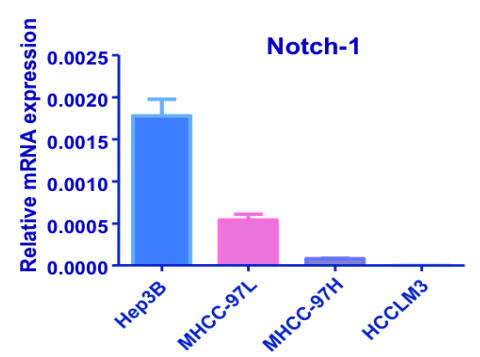

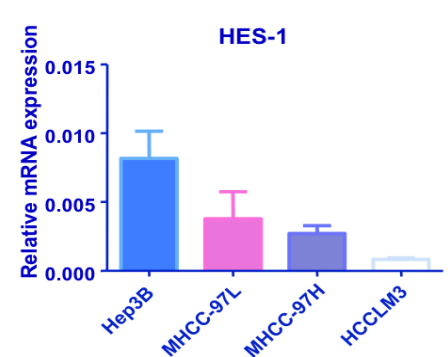
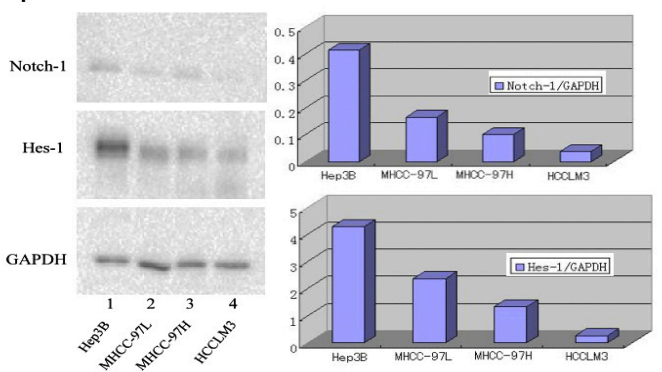

Figure 1. Expression Notch Factors in HCC Cell Lines. (A) Red symbols indicate upregulated genes and blue symbols indicate downregulated genes in MHCC97-L cells as compared with HCCLM3 cells. (B) Upregulated genes (CCND1, HES-1, NOTCH-1, NR4A2) in MHCC97-L cell line. (C) Downregulated genes (FZD1, HOXB4, NOTCH-3, SHH) in MHCC97-L cell line. (D) mRNA expression of Notch-1 in Hep3B, MHCC-97L, MHCC-97H, HCCLM3 cell lines. (E) mRNA expression of Hes1 in Hep3B, MHCC-97L, MHCC-97H, HCCLM3 cell lines. (F) Notch-1 and Hes-1 Protein expressions in Hep3B, MHCC-97L, MHCC-97H, HCCLM3 cell lines by western blot 
A

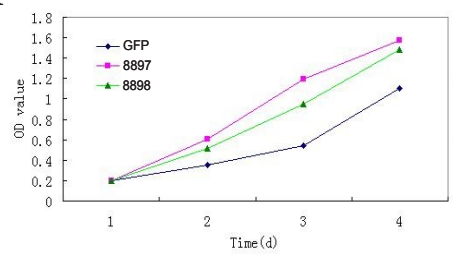

$\mathrm{B}$
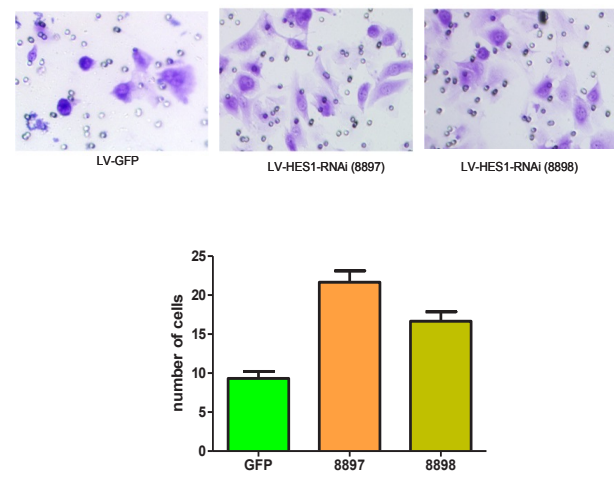

C
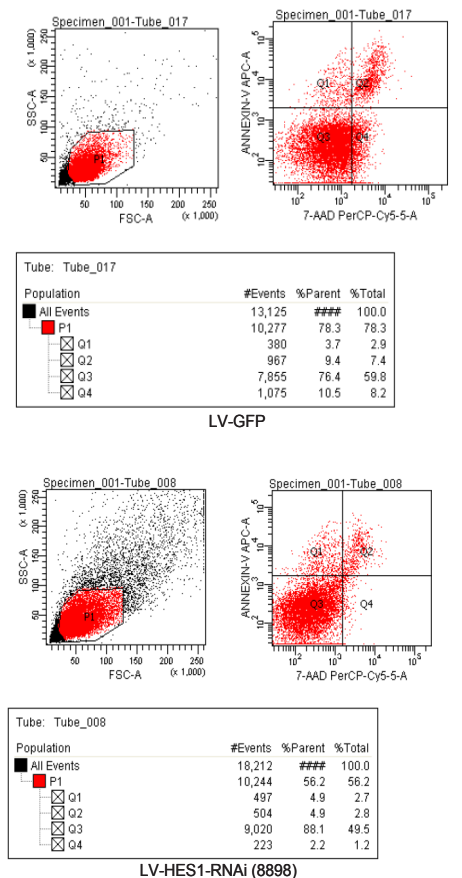
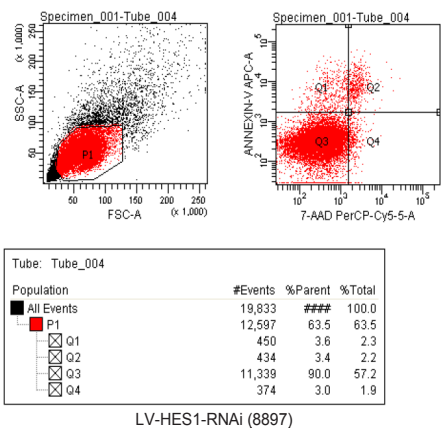

Annexin $\mathrm{V} / \mathrm{PI}$

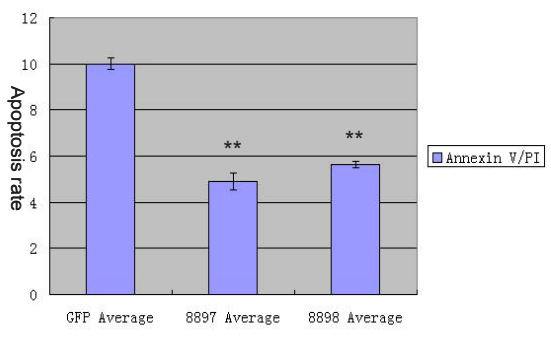

Figure 2. Change of Cell Biological Functions in MHCC-97L Cells by Hes-1 RNAi. (A) Proliferation of MHCC-97L cells was measured following transfection with Hes-1 RNAi plasmid constructs. (B) The invasive potential of MHCC-97L cells was measured $72 \mathrm{~h}$ following transfection of Hes-1 RNAi. ** $p<0.01$ versus control GFP vector. (C) Apoptosis of MHCC-97L cells was measured $72 \mathrm{~h}$ following transfection of Hes-1 RNAi constructs. $* * p<0.01$ versus control GFP vector

proteins quantitative analysis, found that the expression of Notch-1 and Hes-1 in four HCC cell lines (Hep3B, MHCC-97L, MHCC-97H, HCCLM3) decreased as lung metastasis potential increased (Figure 1D-F).

Effect of Hes-1 down-regulation on in vitro proliferation, apoptosis, and invasive activity of $\mathrm{MHCC}-97 \mathrm{~L}$ cells

We used RNAi to silence the Hes-1 gene in MHCC97L cells and observe the effect of Hes-1 down-regulation on HCC cell proliferation, apoptosis, migration and invasion in vitro. GFP expression in cells transfected with shRNA constructs was visualized by fluorescence microscopy $72 \mathrm{~h}$ post-transfection (Supplementary Figure 1A). Both RT-PCR (Supplementary Figure 1B) and western blot (Supplementary Figure 1C) demonstrated that LV-HES1-RNAi (8997) and LV-HES1-RNAi (8998) had superior knockdown efficiency. We therefore used these two constructs for further experiments.

Assessment of cell proliferation identified that proliferation of MHCC-97L cells was enhanced by transfection with LV-HES1-RNAi (8997) and LV-HES1RNAi (8998) (Figure 2 A).

Next, cell invasion was measured using matrigel coated transwell-invasion chambers. The number of migratory MHCC-97L cells significantly increased with transfection of LV-HES1-RNAi (8997) or LV-HES1-RNAi (8998) compared with LV-GFP (Figure $2 \mathrm{~B} ; p<0.01$ for both).

To further elucidate the influence of Hes-1 downregulation in MHCC-97L cells, we measured apoptosis 72 h after transfection with Hes-1 shRNA lentiviral plasmid constructs. Annexin V/PI tests revealed that downregulation of Hes-1 dramatically restrains cell apoptosis $(p<0.01$; Figure 2 C).
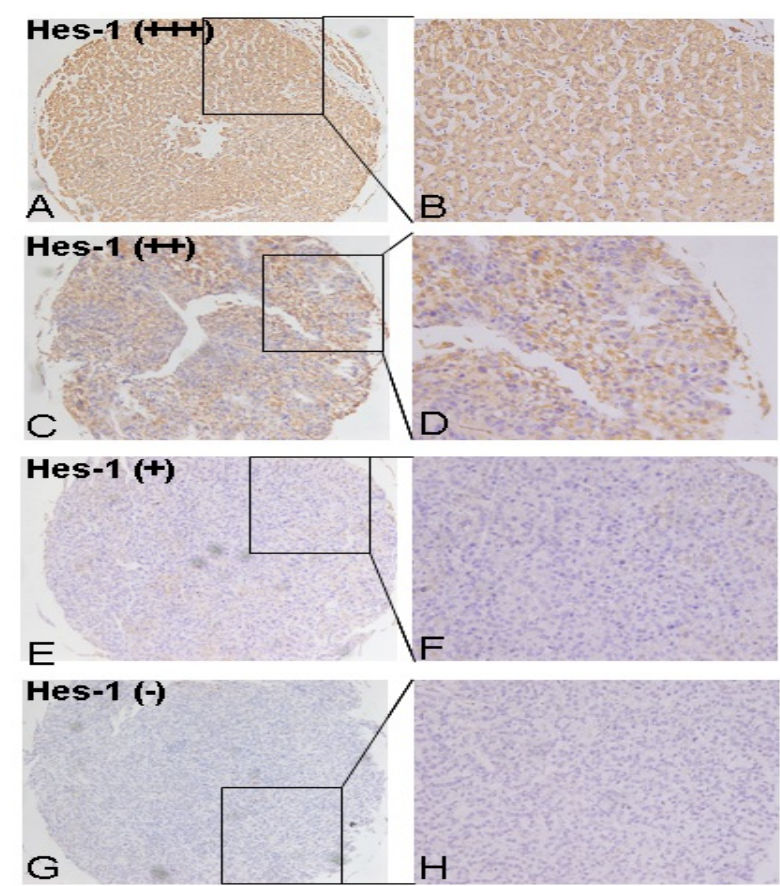

Figure 3. Immunohistochemical Analysis of Hes-1 Protein Expressions in HCC Tissues. Examples of high expression (+++ and ++$)$ and low expression (+ and -$)$. A: Hes-1 $(+++)$, and B: enlarged area of A. C: Hes-1 (++), and D: enlarged area of C. E: Hes-1 (+), and F: enlarged area of E. G: Hes-1 (-), and $\mathrm{H}$ : enlarged of $\mathrm{G}$

Prognostic significance of Notch-1 and Hes-1 in HCC patients

We finally examined the relationship between Hes-1 expression in tissue samples from HCC patients and a variety of clinical parameters. A tissue microarray was constructed using 108 HCC tissues and Hes-1 protein was detected by immunohistochemistry (Figure 3 ). Hes- 1 expression level correlated with tumor number $(p=0.044)$, 

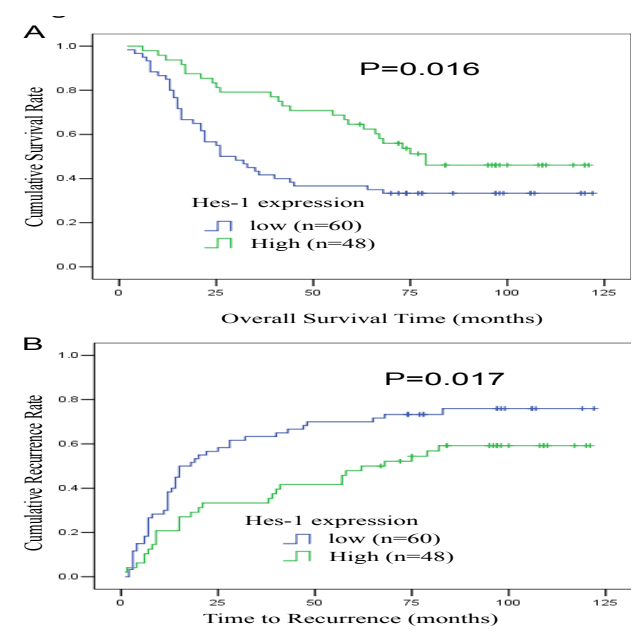

Figure 4. Kaplan-Meier Curves of Survival Analysis among HCC Patients. OS (A) and TTR (B) for low Hes-1 and high Hes- 1 expression in HCC tissue were statistically significant (by the long-rank test)
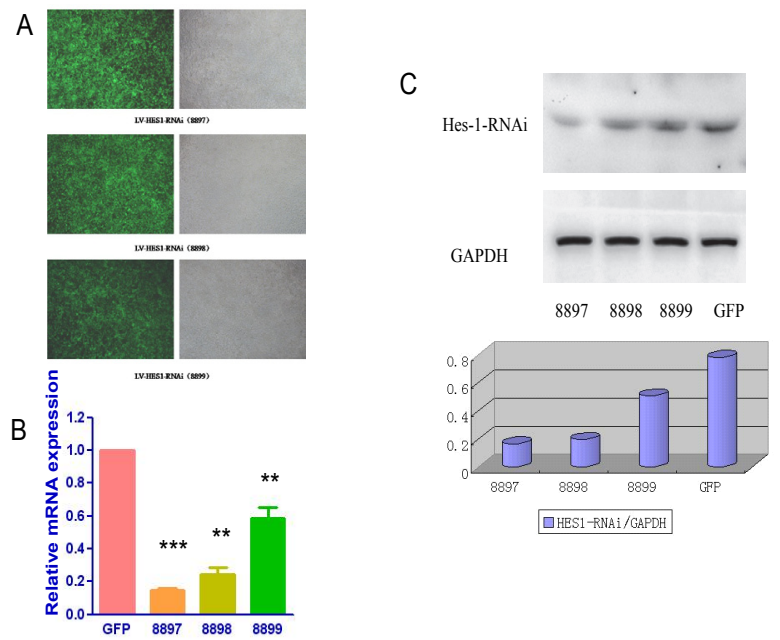

Supplementary Figure 1. Knock-down Efficiency of Three shRNA-Hes-1 Vectors. (A) The transduced cells were visualized by residual GFP expression using. (B) fluorescence microscopy after $72 \mathrm{~h}$ infection of LV-HES1 vectors. (B) Relative mRNA expression of Hes-1 after infect to MHCC-97L cells. (C) Hes-1 protein expression analysis by western blot. $* * * \mathrm{P}<0.001$ and $* *<0.01$ versus control GFP vector
DOI:http://dx.doi.org/10.7314/APJCP.2015.16.9.3811 Prognostic Significance of Hes-1 in Hepatocellular Carcinoma tumor differentiation ( $p=0.001$ ), and serum $\alpha$-fetoprotein (AFP; $p=0.001$ ), while no correlation with age, sex, liver cirrhosis hepatitis B virus antigen, tumor size, vascular invasion, lymphoma metastasis, encapsulation, TNM, or Barcelona Clinic Liver Cancer was observed (all $p>0.05$ ).

The Kaplan-Meier method was used to analyze the relationship of low and high Hes-1 expression with OS and TTR of HCC patients, and expression of Hes-1 in HCC significantly correlated with a favorable prognosis (Figure 4). The median OS time of patients with high Hes-1 expression was significantly higher than that for low Hes-1 expression HCC patients (26 vs 79 months, $p=0.016)$. The time to recurrence time for low Hes- 1 expression in HCC patients was shorter than that for high Hes-1 expression HCC patients (median TTR for low vs high $=15$ vs 62 months, $p=0.017$ ). Further Cox proportional hazard model analysis demonstrated that Hes-1 was an independent prognostic indicator for OS $(p=0.009$, Table 1).

\section{Discussion}

Current research has not described a clear role for the Notch signaling pathway in HCC, and there are some discrepancies between studies. Qi et al. have reported that Notch-1 signaling may negatively influence the development of HCC. They showed that Notch-1 overexpression inhibited HCC cell growth both in vitro and in vivo. Consistently, Notch-1 signaling induces cell cycle arrest and apoptosis in human HCC through regulation of Cyclin A1, Cyclin E, CDK2, phosho-Rb, p21waf/cip1, p53 and JNK (Qi et al., 2003). In contrast, however, Croquelois et al. used a conditional Notch-1 knockout mouse model to investigate the role of Notch-1 signaling in liver cell proliferation and differentiation and showed that deletion of Notch-1 led to continuous proliferation of hepatocytes (Croquelois et al., 2005).

Hes-1 mRNA expression is frequently used as an indicator of Notch signaling activity and Notch-1/Hes-1 activation has been implicated in multiple human cancers (Weng and Aster, 2004; Liu et al., 2007; Kannan et al., 2011). In addition, Ye et. al. reported that siRNA mediated

Table 1. Univariate and Multivariate Analyses of Factors Associated with OS and TTR

\begin{tabular}{|c|c|c|c|c|c|c|c|c|}
\hline \multirow{3}{*}{ Factors } & \multicolumn{5}{|c|}{ OS } & \multirow{2}{*}{\multicolumn{3}{|c|}{$\frac{\text { TTR }}{\text { multivariate }}$}} \\
\hline & \multirow[t]{2}{*}{ univariatep $\mathrm{P}$} & \multicolumn{3}{|c|}{ multivariate } & \multirow[t]{2}{*}{ univariate $\mathrm{P}$} & & & \\
\hline & & HR & $95 \% \mathrm{CI}$ & $\bar{P}$ & & HR & $95 \% \mathrm{CI}$ & $\mathrm{P}$ \\
\hline Age: $\leq 50$ vs $>50$ & 0.877 & & & & 0.931 & & & \\
\hline Sex: male vs female & 0.281 & & & & 0.854 & & & \\
\hline Liver cirrhosis: no vs yes & 0.650 & & & & 0.458 & & & \\
\hline HBsAg: negative vs positive & 0.127 & & & & 0.857 & & & \\
\hline Tumor size: $\leq 5 \mathrm{~cm}$ vs $>5 \mathrm{~cm}$ & 0.419 & & & & 0.330 & & & \\
\hline Tumoe number: single vs multiple & 0.028 & 1.978 & $1.127-3.471$ & 0.017 & 0.051 & & & \\
\hline Vascular invasion: no vs yes & 0.000 & 3.256 & $1.927-5.502$ & 0.000 & 0.000 & 2.609 & $1.584-4.297$ & 0.000 \\
\hline Lymphoma metastasis: no vs yes & 0.000 & 3.150 & $1.014-9.783$ & 0.047 & 0.008 & & & \\
\hline Encapsulation: no vs yes & 0.334 & & & & 0.078 & & & \\
\hline Tumor differentiation: I-II vs III-IV & 0.135 & & & & 0.238 & & & \\
\hline $\operatorname{Serum}$ AFP (ng/ml): $\leq 20$ vs $>20$ & 0.013 & & & & 0.037 & & & \\
\hline TNM: I-II vs III-IV & 0.000 & & & & 0.001 & 2.387 & $1.195-4.771$ & 0.014 \\
\hline Hes-1: low vs high & 0.018 & 0.494 & $0.290-0.840$ & 0.009 & 0.020 & 0.553 & $0.342-0.894$ & 0.016 \\
\hline BCLC: 0 vs A vs B & 0.003 & & & & 0.031 & & & \\
\hline
\end{tabular}


Notch-1 silencing enhances docetaxel induced mitotic arrest and apoptosis in prostate cancer cells (Ye et al., 2012). However, the role of Hes-1 in HCC cell lines and in tissue samples from HCC patients has, to our knowledge, not previously been reported. Our study confirms that a reduction of Notch-1 and Hes-1 expression is present in Hep3B, MHCC-97L, MHCC-97H, and HCCLM3 cells with elevated lung metastasis potentials at both the mRNA and protein levels.

We used RNA interference to downregulate the Hes-1 gene in the MHCC-97L cell line and observed that Hes1 knockdown resulted in increased cell proliferation, inhibited apoptosis, and increased migration and invasion in vitro. This is the first report to explore the influence of downregulation of Hes-1 protein expression on HCC cells, and indeed on any cancer cells. Additionally, the prognostic value of Hes-1 in human HCC has not been previously reported. Mann et al. have previously examined components of the Notch signaling pathway including Notch-1, -3, -4, Hes-1, and Hey-1 in HCC. However, they did not observe any significant association between Hes-1 expression and OS or disease-free survival. In contrast, Kaplan-Meier analysis in our study revealed that the OS rate of HCC patients following surgery was greater in patients with higher Hes-1 expression. Additionally, those patients with high Hes-1 expression were less likely to experience tumor recurrence. Further Cox proportional hazard model analysis also showed that Hes-1 was an independent prognostic indicator for OS and TTR.

In conclusion, Hes- 1 expression is decreased in HCC cell lines that have higher lung metastasis potential. Down-regulation of the Hes-1 gene in MHCC-97L cells resulted in increased cell proliferation, inhibited apoptosis, and increased migration and invasion. Finally, Hes-1 has a potential prognostic values in HCC patients following surgery, and may be an independent prognostic indicator for OS and likelihood of tumor recurrence.

\section{Acknowledgements}

This study was supported by a grant from the State Key Basic Research Program of China (973 Program) (No. 2004CB518708) and by a Zhongshan Hospital Research Grant for Young Researchers.

\section{References}

Beasley RP (1988). Hepatitis B virus. The major etiology of hepatocellular carcinoma. Cancer, 61, 1942-56.

Cantarini MC, de la Monte SM, Pang M, et al (2006). Aspartylasparagyl beta hydroxylase over-expression in human hepatoma is linked to activation of insulin-like growth factor and notch signaling mechanisms. Hepatol, 44, 446-57.

Caporaso N, Romano M, Marmo R, et al (1991). Hepatitis C virus infection is an additive risk factor for development of hepatocellular carcinoma in patients with cirrhosis. $J$ Hepatol, 12, 367-71.

Colombo M (1999). Hepatitis C virus and hepatocellular carcinoma. Semin Liver Dis, 19, 263-9.

Croquelois A, Blindenbacher A, Terracciano L, et al (2005). Inducible inactivation of Notch1 causes nodular regenerative hyperplasia in mice. Hepatol, 41, 487-96.
Dailey DD, Anfinsen KP, Pfaff LE, et al (2013). HES1, a target of Notch signaling, is elevated in canine osteosarcoma, but reduced in the most aggressive tumors. BMC Vet Res, $9,130$.

He J, Gu D, Wu X, et al (2005). Major causes of death among men and women in China. N Engl J Med, 353, 1124-34.

Jin GZ, Yu WL, Dong H, et al (2013). SUOX is a promising diagnostic and prognostic biomarker for hepatocellular carcinoma. J Hepatol, 59, 510-7.

Kannan S, Fang W, Song G, et al (2011). Notch/HES1-mediated PARP1 activation: a cell type-specific mechanism for tumor suppression. Blood, 117, 2891-900.

Kaushik G, Venugopal A, Ramamoorthy P, et al (2014). Honokiol inhibits melanoma stem cells by targeting notch signaling. Mol Carcinog, Dec 9[Epub ahead of print], PMID: 25491779.

Lindsell CE, Shawber CJ, Boulter J, et al (1995). Jagged: a mammalian ligand that activates Notch1. Cell, 80, 909-17.

Liu J, Ye F, Chen H, et al (2007). Expression of differentiation associated protein Hes1 and Hes5 in cervical squamous carcinoma and its precursors. Int J Gynecol Cancer, 17, 1293-9.

Mo QG, Liang AM, Yang NW, et al (2003). [Surgerypredominant comprehensive therapy for 134 patients with small hepatocellular carcinoma]. Ai Zheng, 22, 189-91.

Parkin DM, Bray F, Ferlay J, et al (2005). Global cancer statistics, 2002. CA Cancer J Clin, 55, 74-108.

Portolani N, Coniglio A, Ghidoni S, et al (2006). Early and late recurrence after liver resection for hepatocellular carcinoma: prognostic and therapeutic implications. Ann Surg, 243, 229-35.

Qi R, An H, Yu Y, et al (2003). Notch1 signaling inhibits growth of human hepatocellular carcinoma through induction of cell cycle arrest and apoptosis. Cancer Res, 63, 8323-9.

Ranganathan P, Weaver KL, Capobianco AJ (2011). Notch signalling in solid tumours: a little bit of everything but not all the time. Nat Rev Cancer, 11, 338-51.

Tang Z, Zhou X, Lin Z, et al (1999). Surgical treatment of hepatocellular carcinoma and related basic research with special reference to recurrence and metastasis. Chin Med J (Engl), 112, 887-91.

Tian J, Tang ZY, Ye SL, et al (1999). New human hepatocellular carcinoma (HCC) cell line with highly metastatic potential (MHCC97) and its expressions of the factors associated with metastasis. Br J Cancer, 81, 814-21.

Weng AP, Aster JC (2004). Multiple niches for Notch in cancer: context is everything. Curr Opin Genet Dev, 14, 48-54.

Ye QF, Zhang YC, Peng XQ, et al (2012). siRNA-mediated silencing of Notch-1 enhances docetaxel induced mitotic arrest and apoptosis in prostate cancer cells. Asian Pac J Cancer Prev, 13, 2485-9. 